\title{
APPROXIMATE UNITARY EQUIVALENCE OF CONTINUOUS NESTS
}

\author{
KENNETH R. DAVIDSON ${ }^{1}$
}

ABSTRACT. A short proof is given of an important theorem of N. Andersen: All continuous nests are approximately unitarily equivalent.

The purpose of this note is to give a simple proof of an important theorem of Niels Andersen [1].

THEOREM. Let $\mathcal{N}$ and $\mathcal{M}$ be continuous nests of projections and let $\theta$ be an order isomorphism of $\mathcal{N}$ onto $\mathcal{M}$. Given $\varepsilon>0$, there is a unitary $U$ such that $\theta(N) U-U N$ is compact for all $N$ in $\mathcal{N}$, and $\sup \{\|\theta(N) U-U N\|: N \in \mathcal{N}\}<\varepsilon$.

This theorem is a key ingredient in David Larson's solution of the Ringrose problem [6, 7]: all continuous nests are similar. This in turn led to the author's Similarity Theorem [4]: Suppose $\mathcal{N}$ and $\mathcal{M}$ are nests, and $\theta$ is an order isomorphism of $\mathcal{N}$ onto $\mathcal{M}$ which preserves dimension. Then $\mathcal{N}$ and $\mathcal{M}$ are similar.

This theorem was given a second proof by William Arveson [3]. His proof extended the methods of his earlier paper [2] to give a version of Dan Voiculescu's theorem [8] valid for certain nonseparable $C^{*}$-algebras. Both proofs of the theorem are long and difficult. Our proof is a synthesis of these two proofs which is both shorter and easier. Although this paper is self-contained, a familiarity with [2] would be an asset.

I would like to thank both A. Hopenwasser and the reviewer for some helpful comments on the exposition.

1. Preliminaries. A continuous nest $\mathcal{N}$ is a complete chain of subspaces of a separable Hilbert space which is order isomorphic to $[0,1]$. Let $\left\{N_{t}, 0 \leq t \leq 1\right\}$ be the orthogonal projections onto the elements of the nest. In this note, the nest $\mathcal{N}$ will be routinely identified with the corresponding nest of projections. There is a spectral measure on $[0,1]$ defined by

$$
E(a, b]=N_{b}-N_{a}
$$

and extended in the natural way. One can choose a finite scalar Borel measure on $[0,1]$ which is mutually absolutely continuous with $E$. The support of a vector $x$ is the smallest closed set $C$ such that $E(C) x=x$. A nest $\mathcal{N}$ is cyclic if there is a vector $x$ (a cyclic vector) such that $\operatorname{span}\left\{N_{t} x\right\}$ equals $\nVdash$. A cyclic vector necessarily has support $[0,1]$.

Received by the editors March 8, 1985 and, in revised form, August 5, 1985.

1980 Mathematics Subject Classification. Primary 47D25; Secondary 46L35, 46M20.

Key words and phrases. Approximate unitary equivalence, nests, compact perturbations.

${ }^{1}$ Research partially supported by a grant from NSERC, Canada. The author would like to thank Arizona State University for its hospitality during the period that this work was done. He also wishes to thank D. Herrero for typing the original draft. 
The study of triangular algebras was initiated by Kadison and Singer [5], and it follows from their Theorem 3.3.1 and its proof that any continuous nest $N$ with a cyclic vector can be reparametrized to be unitarily equivalent to the Volterra nest $\mathcal{N}_{0}$ on $L^{2}(0,1)$ consisting of subspaces $N_{t}$ of functions supported in $[0, t]$. Although we will not need this result, certain computations carried out in our proof are easier for this particular nest (see remarks in §6). The reader might well keep this example in mind as he reads the proof.

If $\mathcal{N}=\left\{N_{t}\right\}$ and $\mathcal{M}=\left\{M_{t}\right\}$ are two nests with given parametrizations on $[0,1]$, define $\mathcal{N} \oplus \mathcal{M}=\left\{N_{t} \oplus M_{t} ; 0 \leq t \leq 1\right\}$. It will be convenient to allow redundancy in the parametrization, in that $N_{t_{0}}=N_{t_{1}}$ for $t_{0}<t_{1}$ is allowed. So, if $\mathcal{N}=\left\{N_{t}\right\}$ is a nest and $P$ is a projection commuting with $\mathcal{N}$, then $\mathcal{N}_{1}=\mathcal{N} \mid P \mathcal{H}$ and $\mathcal{N}_{2}=\mathcal{N} \mid P^{\perp} \forall$ are nests with the induced parametrizations. (In this case, redundant parametrization is natural.) It is clear that $\mathcal{N}=\mathcal{N}_{1} \oplus \mathcal{N}_{2}$, and that every decomposition of $\mathcal{N}$ into direct summands occurs in this way. Define the support of a summand $\mathcal{N}_{1}$ (relative to $\mathcal{N}$ ) to be the closure of the union $\{\operatorname{supp}(x): x \in P \mathscr{K}\}$. Note that if $\mathcal{N}_{1}$ has a cyclic vector $x_{1}$, then $\operatorname{supp}\left(x_{1}\right)=\operatorname{supp}\left(\mathcal{N}_{1}\right)$.

If $\theta$ is an order isomorphism between $\mathcal{N}$ and another continuous nest $\mathcal{M}$, then $\mathcal{M}$ can be parametrized as $\left\{M_{t}, 0 \leq t \leq 1\right\}$ so that $\theta\left(N_{t}\right)=M_{t}$. Two such nests will be called $\varepsilon$-unitarily equivalent $\left(\mathcal{N} \sim_{\varepsilon} \mathcal{M}\right)$ if there is a unitary $U$ such that $M_{t} U-U N_{t}$ is compact for $0 \leq t \leq 1$, and

$$
\sup _{0 \leq t \leq 1}\left\|M_{t} U-U N_{t}\right\|<\varepsilon .
$$

Two nests are approximately unitarily equivalent $\left(\mathcal{N} \sim_{a} \mathcal{M}\right)$ provided $\mathcal{N} \sim_{\varepsilon} \mathcal{M}$ for every $\varepsilon>0$.

A rank one operator will be denoted as $x \otimes y^{*}$, which acts by the formula $x \otimes$ $y^{*}(z)=(z, y) x$. Note that $A\left(x \otimes y^{*}\right) B=A x \otimes\left(B^{*} y\right)^{*}$.

2. Approximate infinite multiplicity. The purpose of this section is to show that every continuous nest is the infinite direct sum of cyclic nests with full support. In a certain sense, this indicates that all continuous nests behave as if they have infinite multiplicity.

LEMMA 1. Let $\mathcal{N}$ be a continuous nest, and let $J_{i}$ be open subsets of $J_{0}=(0,1)$, $i \geq 1$. Then $\mathcal{N} \simeq \sum_{i=0}^{\infty} \bigoplus \mathcal{N}_{i}$ where each $\mathcal{N}_{i}$ is a continuous nest with support $\bar{J}_{i}$. If $\mathcal{N}$ is cyclic, then each $\mathcal{N}_{i}$ is cyclic.

PROOF. Let $\mu$ be a finite regular Borel measure on $[0,1]$ mutually absolutely continuous with the spectral measure $E$ of $\mathcal{N}$. Since $\mu$ is nonatomic, there are pairwise disjoint measurable sets $A_{i}$ included in $J_{i}$ with $(0,1)=\bigcup_{i=0}^{\infty} A_{i}$ and $\mu\left(A_{i} \cap I\right)>0$ for every open subset $I$ of $J_{i}$. Set $\mathcal{N}_{i}=\mathcal{N} \mid E\left(A_{i}\right) \mathcal{H}$. It is readily verified that the support of $\mathcal{N}_{i}$ is $\bar{A}_{i}=\bar{J}_{i}$, and $\mathcal{N} \simeq \sum_{i=0}^{\infty} \bigoplus \mathcal{N}_{i}$. If $x$ is a cyclic vector $\mathcal{N}$, then $x_{i}=E\left(A_{i}\right) x$ is cyclic for each $\mathcal{N}_{i}$.

LEMMA 2. Let $\mathcal{N}$ be a continuous nest. Then $\mathcal{N} \simeq \sum_{i=1}^{\infty} \bigoplus \mathcal{N}_{i}$ where each $\mathcal{N}_{i}$ has a cyclic vector $x_{i}$ with support $[0,1]$.

PROOF. First, note several simple facts about cyclic vectors. If $\mathcal{N}$ has a cyclic vector $x$, and $P$ commutes with $\mathcal{N}$, then $P x$ is cyclic for $\mathcal{N} \mid P \mathscr{H}$. Also, if $\mathcal{N}_{i}$ have cyclic vectors $x_{i}, i=1$ and 2 , with supports $S_{i}$ such that $E\left(S_{1} \cap S_{2}\right)=0$, then 
$x_{1} \oplus x_{2}$ is a cyclic vector for $\mathcal{N}_{1} \oplus N_{2}$. Suppose $\mathcal{N}$ is a nest and $x \neq 0$ is a vector. Let $H_{x}$ be the closed span of $\left\{N_{t} x: 0 \leq t \leq 1\right\}$. Clearly, the projection onto $H_{x}$ commutes with $\mathcal{N}$, and $\mathcal{N}_{x}=\mathcal{N} \mid \mathfrak{H}_{x}$ is a summand of $\mathcal{N}$ with cyclic vector $x$ and support equal the support of $x$.

Start with a vector $x_{0}$ with support $[0,1]$, and form the cyclic nest $\left(\mathcal{N}_{x_{0}}, x_{0}, \mathfrak{H}_{x_{0}}\right)$. This has support $[0,1]$. Using Zorn's Lemma, one can extend this to a maximal family of pairwise orthogonal cyclic symmands $\left\{\left(\mathcal{N}_{x_{\alpha}}, x_{\alpha}, \mathscr{H}_{x_{\alpha}}\right)\right\}$. It follows readily that $\mathcal{H}=\sum_{\alpha} \bigoplus \mathfrak{H}_{x_{\alpha}}$ and $\mathcal{N}=\sum_{\alpha} \bigoplus \mathcal{N}_{x_{\alpha}}$. Of course, supp $\left(x_{\alpha}\right)=C_{\alpha}$ need not be all of $[0,1]$ except for $\alpha=0$. This is remedied by using Lemma 1 to attach to each $\mathcal{N}_{x_{\alpha}}, \alpha \neq 0$, a summand of $\mathcal{N}_{x_{0}}$.

Let $J_{\alpha}$ be the complement of $C_{\alpha}$ in $[0,1]$. By Lemma 1 , the cyclic nest $\mathcal{N}_{x_{0}}$ can be decomposed as a direct sum $\sum_{\alpha} \oplus \mathcal{M}_{\alpha} \oplus \sum_{i=0}^{\infty} \mathcal{N}_{i}$ so that supp $\mathcal{M}_{\alpha}=\bar{J}_{\alpha}$ and $\operatorname{supp} \mathcal{N}_{i}=[0,1]$. Furthermore, $\mathcal{M}_{\alpha}$ has a cyclic vector $y_{\alpha}$ such that $E\left(C_{\alpha}\right) y_{\alpha}=0$ and $\mathcal{N}_{i}$ has a cyclic vector $z_{i}$. From the first paragraph of the proof, it follows that $\mathcal{N}_{x_{\alpha}} \oplus \mathcal{M}_{\alpha}$ has a cyclic vector $x_{\alpha} \oplus y_{\alpha}$ and support $[0,1]$ for $\alpha \neq 0$. Thus, $\mathcal{N}$ decomposes as

$$
\mathcal{N} \cong \sum_{\alpha \neq 0}\left(\mathcal{N}_{x_{\alpha}} \oplus \mathcal{M}_{\alpha}\right) \oplus \sum_{i=1}^{\infty} \mathcal{N}_{i}
$$

as a sum of cyclic nests of support $[0,1]$.

3. A quasicentral approximate unit. Let $\mathcal{N}$ be a continuous nest with cyclic vector $x$. For each $N \geq 1$, let $P_{k, N}=E\left((k-1) / 2^{N}, k / 2^{N}\right)$ and $x_{k, N}=P_{k, N} x$. Let $P_{N}$ be the orthogonal projection onto the span of $\left\{x_{k, N}, 1 \leq k \leq 2^{N}\right\}$. Since $x$ is cyclic, the union of this increasing sequence of subspaces is dense in $k$.

LEMMA 3. Given an integer $N \geq 1$ and an $\varepsilon>0$, there is a finite rank contraction $E \geq P_{N}$ which is a convex combination of $\left\{P_{n}, n \geq N\right\}$ such that $\sup _{0 \leq t \leq 1}\left\|N_{t} E-E N_{t}\right\|<\varepsilon$.

ProOF. Let $f(t)=\left\|N_{t} x\right\|^{2}$, and note that this is a strictly increasing continuous function on $[0,1]$. From the uniform continuity of $f$, one obtains

$$
\lim _{N \rightarrow \infty} \max _{1 \leq k \leq 2^{N}}\left\|x_{k, N}\right\|=0 \text {. }
$$

Let $\bar{x}_{k, N}=\left\|x_{k, N}\right\|^{-1} x_{k, N}$. Then for $M=N+n$, one can write

$$
\bar{x}_{k, N}=\sum_{i=(k-1) 2^{n}+1}^{k 2^{n}} a_{i} \bar{x}_{i, M} .
$$

A computation shows that $a_{i}=\left\|x_{k, N}\right\|^{-1}\left\|x_{i, M}\right\|$, so one obtains that

tends to 0 as $M$ tends to $\infty$.

$$
\delta(N, M)=\max _{1 \leq i \leq 2^{M}} a_{i}
$$

Let $L=\left[\varepsilon^{-1}+1\right]$. Choose integers $N=M_{1}<M_{2}<\cdots<M_{L}$ such that $\delta\left(M_{i}, M_{i+1}\right)<1 / 2 L^{2}$ for $1 \leq i \leq L$. Define $E=L^{-1} \sum_{i=1}^{L} P_{i}$. Fix $t$ in $[0,1]$, and consider $\left[N_{t}, E\right]=N_{t} E-E N_{t}$. For each $i$, there is an integer $k_{i}$ so that $k_{i}-1 \leq 2^{M_{i}} t \leq k_{i}$. Since

$$
P_{M_{i}}=\sum_{k=1}^{2^{M_{i}}} \bar{x}_{k, M_{i}} \otimes \bar{x}_{k, M_{i}}^{*}
$$


and the $\bar{x}_{k, M_{i}}$ have disjoint supports, one obtains that

$$
\left[N_{t}, P_{M_{i}}\right]=\left[N_{t}, \bar{x}_{k_{i}, M_{i}} \otimes \bar{x}_{k_{i}, M_{i}}^{*}\right]=x_{i} \otimes y_{i}^{*}-y_{i} \otimes x_{i}^{*}
$$

where $x_{i}=N_{t} \bar{x}_{k_{i}, M_{i}}$ and $y_{i}=N_{t}^{\perp} \bar{x}_{k_{i}, M_{i}}$. So

$$
\left[N_{t}, E\right]=L^{-1} \sum_{i=1}^{L} x_{i} \otimes y_{i}^{*}-y_{i} \otimes x_{i}^{*} .
$$

Note that if $i<j$, then

$$
\left|\left(x_{i}, x_{j}\right)\right|+\left|\left(y_{i}, y_{j}\right)\right|=\left(\bar{x}_{k_{i}, M_{i}}, \bar{x}_{k_{j}, M_{j}}\right) \leq \delta\left(M_{i}, M_{j}\right)<1 / 2 L^{2} .
$$

As this means that $\left\{x_{i}, y_{i}: 1 \leq i \leq L\right\}$ are almost orthogonal, a routine estimate yields

$$
\left\|\left[N_{t}, E\right]\right\| \leq L^{-1}\left(\max \left\|x_{i} \otimes y_{i}^{*}\right\|+L^{2}\left(1 / 2 L^{2}\right)\right)<\varepsilon\left(\frac{1}{2}+\frac{1}{2}\right)=\varepsilon .
$$

4. Approximate embeddings. Let $\mathcal{N}$ and $\mathcal{M}$ be continuous nests with cyclic vectors $x$ and $y$ respectively. Let $\bar{x}_{k, N}\left(\bar{y}_{k, N}\right), P_{k, N}\left(Q_{k, N}\right), P_{N}\left(Q_{N}\right)$ be as in the previous section.

LEMMA 4. Given an integer $N_{0}$ and an $\varepsilon>0$, there is a unitary $U$ satisfying

$$
\sup _{0 \leq t \leq 1}\left\|\left(M_{t} U-U N_{t}\right) P_{N_{0}}\right\|<\varepsilon .
$$

Proof. For $N>N_{0}$, define a unitary $U_{N}$ by setting $U_{N} \bar{x}_{k, N}=\bar{y}_{k, N}, 1 \leq k \leq$ $2^{N}$, and extending it in such a way that $U P_{k, N} \nVdash=Q_{k, N} \nvdash$. Fix $t$ in $[0,1]$, and find integers $k$ and $i_{o}$ so that

$$
k-1 \leq 2^{N_{0}} t \leq k \quad \text { and } \quad i_{0}-1 \leq 2^{N} t \leq i_{0} .
$$

Then if $\bar{x}_{k, N_{0}}=\sum a_{i} \bar{x}_{i, N}$, one has

$$
\begin{aligned}
\left\|\left(M_{t} U_{N}-U_{N} N_{t}\right) P_{N_{0}}\right\| & =\left\|\left(M_{t} U_{N}-U_{N} N_{t}\right) \bar{x}_{k, N_{0}}\right\| \\
& =\left|a_{i_{0}}\right| \cdot\left\|M_{t} \bar{y}_{i_{0}, N}-U_{N} N_{t} \bar{x}_{i_{0}, N}\right\| \leq 2 \delta\left(N_{0}, N\right) .
\end{aligned}
$$

For $N$ sufficiently large,

$$
\sup _{0 \leq t \leq 1}\left\|\left(M_{t} U_{N}-U_{N} N_{t}\right) P_{N_{0}}\right\| \leq 2 \delta\left(N_{0}, N\right)<\varepsilon .
$$

Now proceed as in Arveson's proof of Voiculescu's theorem [3].

LEMma 5. Let $\mathcal{N}$ and $\mathcal{M}$ be continuous nests, and let $\varepsilon>0$. There is an isometry $U$ of $H^{(\infty)}$ into $H$ such that $M_{t} U-U N_{t}^{(\infty)}$ is compact for $0 \leq t \leq 1$, and

$$
\sup _{0 \leq t \leq 1}\left\|M_{t} U-U N_{t}^{(\infty)}\right\|<\varepsilon .
$$

ProOF. We will make use of the following fact: If $E$ is positive and $\|X\| \leq$ 1, then $\left\|X E^{1 / 2}-E^{1 / 2} X\right\| \leq 7\|X E-E X\|^{1 / 2}$. (The weaker version in [2] will suffice.) By Lemma 2, $\mathcal{M}$ can be decomposed as $\sum_{i} \sum_{j} \bigoplus \mathcal{M}_{i j}$ where $\mathcal{M}_{i j}$ are continuous nests on $\mathcal{M}_{i j}$ with cyclic vector $y_{i j}$ of support $[0,1]$. Similarly, $\mathcal{N}^{(\infty)}$ can be decomposed as $\sum_{i} \bigoplus \mathcal{N}_{i}$ where $\mathcal{N}_{i}$ act on $K_{i}$ and have cyclic vectors $x_{i}$ of support $[0,1]$. So it suffices to provide an isometry $V$ of $K_{i}$ into $\sum_{j} \bigoplus H_{i j}$ such that 
$M_{t} V-V N_{t} \mid K_{i}$ is the compact of norm less than $\varepsilon_{i}=2^{-i} \varepsilon$. For convenience, the subscript $i$ and restriction to $K_{i}$ will be dropped from the notation.

Recursively, choose integers $N_{k}$, contractions $E_{k}$, and unitaries $U_{k}$ taking $K_{i}$ onto $\mathfrak{H}_{i k}$ such that $N_{0}=0, E_{0}=0$, and

(i) $P_{N_{k}} \leq E_{k} \leq P_{N_{k+1}}$

(ii) $\sup _{0 \leq t \leq 1}\left\|N_{t} E_{k}-E_{k} N_{t}\right\|<2^{-8}\left(\varepsilon / 2^{k+1}\right)^{2}$

(iii) $\sup _{0 \leq t \leq 1}\left\|\left(M_{t} U_{k}-U_{k} N_{t}\right) P_{N_{k+1}}\right\|<2^{-k-1} \varepsilon$.

Let $N_{1}=1$. Given $N_{k}, E_{k-1}$ and $U_{k-1}$, use Lemma 3 to obtain $E_{k}$ satisfying (i) and (ii) and choose $N_{k+1}$ accordingly. Then use Lemma 4 to provide $U_{k}$. Set $F_{k}=\left(E_{k}-E_{k-1}\right)^{1 / 2}$ and $V=\sum_{k=1}^{\infty} U_{k} F_{k}$.

Since the $U_{k}$ have the orthogonal ranges $V^{*} V=\sum_{k=1}^{\infty} F_{k}^{2}=I$, and thus $V$ is an isometry. Also

$$
\begin{aligned}
\left\|F_{k} N_{t}-N_{t} F_{k}\right\| & \leq 7\left\|F_{k}^{2} N_{t}-N_{t} F_{k}^{2}\right\|^{1 / 2} \\
& \leq 7 \cdot 2^{-4}\left[\left(\varepsilon / 2^{k+1}\right)^{2}+\left(\varepsilon / 2^{k+1}\right)^{2}\right]^{1 / 2}<\varepsilon / 2^{k+1} .
\end{aligned}
$$

Thus

$$
\begin{aligned}
\left\|M_{t} V-V N_{t}\right\| & =\left\|\sum_{k=1}^{\infty}\left(M_{t} U_{k}-U_{k} N_{t}\right) F_{k}+U_{k}\left(N_{t} F_{k}-F_{k} N_{t}\right)\right\| \\
& \leq \sum_{k=1}^{\infty}\left\|\left(M_{t} U_{k}-U_{k} N_{t}\right) P_{N_{k}+1}\right\|+\left\|N_{t} F_{k}-F_{k} N_{t}\right\| \\
& <\sum_{k=1}^{\infty} 2^{-k-1} \varepsilon+2^{-k-1} \varepsilon=\varepsilon .
\end{aligned}
$$

As each term is finite rank, the sum $M_{t} V-V N_{t}$ is compact.

5. Proof of the Theorem. Let $\mathcal{N}$ and $\mathcal{M}$ be continuous nests, and let $U$ be the isometry provided by Lemma 5 . Let $P=U U^{*}$, and compute:

$$
M_{t} P-P M_{t}=\left(M_{t} U-U N_{t}^{(\infty)}\right) U^{*}-U\left(M_{t} U-U N_{t}^{(\infty)}\right)^{*} .
$$

Thus $M_{t} P-P M_{t}$ is compact of norm at most $2 \varepsilon$ for all $0 \leq t \leq 1$. Let $\mathcal{L}$ be the compression of $\mathcal{M}$ to $P^{\perp} \mathcal{H}$, and suppose that this is a continuous nest. Then the unitary $W$ of $P^{\perp} \mathcal{H} \oplus \mathcal{H}^{(\infty)}$ onto $\mathcal{H}$ given by $W=P^{\perp} \oplus U$ makes $M_{t} W-W\left(L_{t} \oplus N_{t}^{(\infty)}\right)$ compact of norm at most $3 \varepsilon$. That is,

$$
\mathcal{M} \sim_{3 \varepsilon} \quad \mathcal{L} \oplus \mathcal{N}^{(\infty)} \simeq\left(\mathcal{L} \oplus \mathcal{N}^{(\infty)}\right) \oplus \mathcal{N} \sim_{3 \varepsilon} \mathcal{M} \oplus \mathcal{N} .
$$

Similarly, $\mathcal{N} \sim_{6 \varepsilon} \mathcal{N} \oplus \mathcal{M}$, whence $N \sim_{12 \varepsilon} \mathcal{M}$. As $\varepsilon>0$ is arbitrary, $\mathcal{N}$ and $\mathcal{M}$ are approximately unitarily equivalent.

Unfortunately, I do not know if $\mathcal{L}$ is a continuous nest. However, the formal manipulation is valid. To see this, let $S$ be the shift on $\mathcal{H}^{(\infty)}$ given by $S\left(x_{n}\right)=$ $\left(0, x_{1}, x_{2}, \ldots\right)$ and let $J$ be the injection of $\nvdash$ into $\not(\infty)$ by $J x=(x, 0,0, \ldots)$. Define a unitary $W$ of $\mathscr{H} \oplus \forall$ into $\nvdash$ by $W=\left(P^{\perp}+U S U^{*} U J\right)$. The three approximate equivalences in the previous paragraph come from the factorization of $W$ as

$$
\left(P^{\perp} U\right)\left(\begin{array}{ccc}
P^{\perp} & 0 & 0 \\
0 & S & J
\end{array}\right)\left(\begin{array}{cc}
P^{\perp} & 0 \\
U^{*} & 0 \\
0 & I
\end{array}\right)
$$




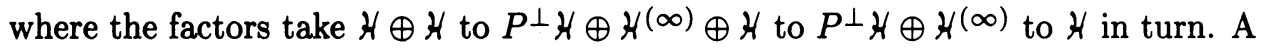
routine computation yields $\mathcal{M} \sim_{6 \varepsilon} \mathcal{M} \oplus \mathcal{N}$ as desired.

6. Remarks. In this section, an attempt will be made to make the constructions more concrete. Consider the Volterra nest $N_{0}$ on $L^{2}(0,1)$ given by projections $N_{t}$ onto $L^{2}(0, t), 0 \leq t \leq 1$. After a change of parametrization, every cyclic continuous nest is unitarily equivalent to $\mathcal{N}_{0}[5]$. In embedding $\mathcal{N}$ into $\mathcal{M}$, the parametrization of one, say $\mathcal{N}$, is arbitrary. So we may assume that it is $\mathcal{N}_{0}$. Now $\mathcal{N}_{0}$ has cyclic vector $x_{0} \equiv 1$. It is easy to compute the function $\delta(N, M)$ in this case: $\delta(N, M)=$ $2^{-(M-N) / 2}$.

Let $E_{n}=2^{-n} \sum_{k=1}^{2^{N}} P_{k n}$. Then the estimates of Lemma 3 yield $\left\|\left[N_{t}, E\right]\right\|<2^{-n}$ for $0 \leq t \leq 1$. Next consider the unitary constructed in Lemma 4 . Since $\mathcal{M}$ is cyclic (with cyclic vector $y$ ), it is unitarily equivalent [5] to the nest of projections $M_{t}$ onto functions supported in $[0, t]$ in $L^{2}(\mu)$ where $\mu$ is a nonatomic measure with support $[0,1]$. The proof of Lemma 4 requires that $U$ take $L^{2}\left((k-1) / 2^{n}, k / 2^{n}\right)$ onto $Q_{k, N} \forall=L^{2}\left(\left((k-1) / 2^{n}, k / 2^{n}\right), \mu\right)$. Further, $U$ takes $x_{k, N}$ (the characteristic function of $\left.\left((k-1) / 2^{N}, k / 2^{N}\right)\right)$ to the cyclic vector $2^{-N / 2} \bar{y}_{k, N}$. This is accomplished explicitly as follows. Let

$$
f(t)=\left\|M_{t} 2^{-N / 2} \bar{y}_{k, N}\right\|^{2}+(k-1) 2^{-N}, \quad(k-1) 2^{-N} \leq t \leq k 2^{-N} .
$$

Note that $f$ is strictly increasing on $[0,1]$ and $f\left(k 2^{-N}\right)=k 2^{-N}$ for $0 \leq k \leq 2^{N}$. For $h$ in $L^{2}\left((k-1) / 2^{N}, k / 2^{N}\right)$, define $U$ by $U h=(h \circ f) 2^{-N / 2} \bar{y}_{k, N}$. Then $U$ is the required unitary since

$$
\|U h\|^{2}=\int|h \circ f|^{2} 2^{-N}\left|\bar{y}_{k, N}\right|^{2} d \mu(t)=\int|h \circ f|^{2} d f(t)=\int|h|^{2} d t=\|h\|^{2} .
$$

So, in principle, one can write down unitaries that embed $\mathcal{N}_{0}$ into the canonical nest $\mathcal{M}$ onto $L^{2}(\mu)^{(\infty)}$, thus producing an approximate summand of $\mathcal{M}$ which is mutually singular. Similarly, by choosing explicit measurable sets to implement Lemma 1 , one can split $\mathcal{N}_{0}$ into a sum of concrete continuous nests. Thus one can write down a unitary which embeds $\mathcal{N}_{0}^{(\infty)}$ into $\mathcal{N}_{0}$. However, it does not seem that these "explicit" unitaries are of a simple enough form to yield further insight.

\section{REFERENCES}

1. M. T. Andersen, Compact perturbations of reflexive algebras, J. Funct. Anal. 38 (1980), 366-400.

2. W. B. Arveson, Notes on extensions of $C^{*}$-algebras, Duke Math. J. 44 (1977), 329-355.

3. __ Perturbation theory for groups and lattices, J. Funct. Anal. 53 (1983), 22-73.

4. K. R. Davidson, Similarity and compact perturbations of nest algebras, J. Reine Angew. Math. 348 (1984), 72-87.

5. R. V. Kadison and I. M. Singer, Triangular operator algebras, Amer. J. Math. 82 (1960), 227-259.

6. D. Larson, A solution to a problem of J. R. Ringrose, Bull. Amer. Math. Soc. (N.S.) 7 (1982), 243-246.

7. , Nest algebras and similarity transformations, Ann. of Math. 121 (1985), 409-427.

8. D. Voiculescu, A non-commutative Weyl-von Neumann theorem, Rev. Roumaine Math. Pures Appl. 21 (1976), 97-113.

Department of Pure Mathematics, University of WATERloo, Waterloo, ONTARIO N2L 3G1, CANADA 